\title{
Artificial Intelligence Perspective on Healthcare
}

\author{
Rehab A. Rayan \\ (High Institute of Public Health, Alexandria University, Alexandria, Egypt \\ rayanr@alexu.edu.eg)
}

\begin{abstract}
Progress in computational sciences for cleaning, sorting, combining, digging, visualizing and managing data along with technological advancements in medical devices have urged needs for further extensive and consistent approaches to discuss the common key issues in medicine and health. Artificial Intelligence (AI) has significantly obtained grounds in everyday living in the era of information technology and it has now landed in healthcare. AI studies' in healthcare are evolving swiftly. However, it could only be the start of observing how it will influence patient care. AI tries to simulate human cognitive capacities. It is carrying a transformation pattern to healthcare, strengthened by the escalating availability of clinical data and sped up advancement in analytics systems. Nonetheless, there is a similar doubt, including some pressing warning at these elevated anticipations. This review examines the present state of AI applications in health, major developments in health AI, and the disparate consequences of health $\mathrm{AI}$ and offers some directions for institutions and caregivers utilizing $\mathrm{AI}$ techniques.
\end{abstract}

Keywords: Information technology, Artificial intelligence, Evidence-based practice, Health, Medicine

\section{Introduction}

Practicing healthcare has been based on collecting sufficient data on the patient's health plus using it to reach conclusions. Medical caregivers ought to depend on their knowledge, understanding, and problem-solving abilities while working with simple means in confined resources. Recently, healthcare has dramatically evolved and now more focus is added upon adopting novel methodologies to avoid illnesses, detect the probability for diseases and intervene to manage the predicted diseases. Likewise, employing technology enables healthcare-givers to render a great deal of competent medical services [Ginsburg and McCarthy 2001]. By the cultural transformation described as digital health, disruptive technologies have offered superior means open for both health practitioners and their cases. These technologies like wearable sensors, Artificial Intelligence (AI), biotechnology or genomics are increasingly heading to producing an immense quantity of data, which need high-level analytics and are making patients the centre-of-care. Rather, producing medicines for populations and taking likewise therapeutic judgments based on some related physical features between subjects, healthcare has moved to prevention, personalization, and precision. Moreover, AI is a fundamental technology that can afford this possibility for daily work. 


\section{Healthcare Practice}

Earlier, medical practice has been directed to finding comprehensive answers, which can handle the highest amount of cases with similar manifestations. For instance, if cough syrup was satisfactory to treat the bulk of the coughing patients and just some cases had dermatitis as a hypersensitivity response to it, sore throat- without questioning- would be managed by cough syrup. Practical proof and gathering experience on an aggregated base was the employed approach of the healthcare society following Hippocrates until about the start of the twentieth century. Following the advances in diagnostic devices, discovering bacteria and viruses, evolving novel medicines and therapeutic techniques, all have made the medical practice to run through complete innovations. The experience-based and 'trial-and-error' strategy in healthcare have created the foundation of evidence-based practice. Respectively, medical professionals ordered diagnostics and medications based on their demonstrated effectiveness in clinical research and scientific articles. For instance, they described in-details the reasons for a sore throat to be adequately tackled by cough syrup and examined the adverse reactions to it. Patients with a hypersensitivity response shall understand that they should shift into an alternative medication. Healthcare practice was confronted by disruptive technologies [Elenko, Underwood and Zohar 2015]. Superior biotechnology, genome sequencing, wearable health sensors, and the data concerning patients' course within healthcare acquired by mobile devices have been generating an immense mass of data. Besides digital health which is manifested by health tracker and the smartphone revolutions [Meskó, Drobni, Bényei, Gergely and Győrffy 2017], it has grown unlikely for a medical practitioner to investigate all these data or to stay updated.

\section{Health AI}

Evidence-based medical practice implies making enlightened clinical decisions via perspectives from previous data. Typically, statistics have encountered this job through denoting data trends by mathematical formulas such as the best-fit line indicated by linear regression. By machine learning (ML), AI offers applications to reveal compound relationships that might not be readily simplified into formulas. For instance, neural networks resemble the human brain in portraying data via huge amounts of interrelated neurons. This permit ML technique to mimic medical practitioners in addressing the complicated problem solving through cautiously considering proof to make valid decisions. Nevertheless, beyond only one professional, these technologies may discover concurrently and operate quickly on an immense feeds' quantity. For instance, today in North London, an AI-driven smartphone application manages competently to triage over one million persons to Accident \& Emergency [Burgess 2017]. Moreover, these techniques can pick up from every progressive event and be open, in no time, to further conditions than a practitioner might check in several time-spans. Consequently, an AI-driven technology has shown to outpace dermatology specialists in sorting strange dermatological injuries accurately or act reliably in frequently rejected jobs by 
professionals, like using chest radiographs to detect pulmonary tuberculosis [Esteva et al. 2017, Lakhani and Sundaram 2017].

\subsection{Public Health Intelligence}

Community health experts and officials accumulate data from numerous origins and study them collectively to figure out the incidence and prevalence of various medical conditions associated hazards. In order to introduce a lucid picture of community health [Shaban-Nejad, Lavigne, Okhmatovskaia and Buckeridge 2017], AI approaches elicit medical and non-medical data at several categories, reconcile and incorporate information around communities with proof regarding the epidemiology and management of non-communicable conditions. Several components, which frame the personal, and public health and welfare, hold origins extrinsic to the traditional medical arrangement. Modern evolutions in AI techniques allow multi-dimensional designing to merge personal data with social markers, the quantifiable indices of societal conditions, at the population-level to enhance anticipating and surveying diseases and executing and rating public health initiatives. For instance, societal health determinants may forecast and discover childhood asthma cases prone to physician revisits [Shin, Mahajan, Akbilgic and Shaban-Nejad 2018].

Additionally, growing mobile networks and the prominence of wearable devices along with the rise in solutions like health IoT (utilizing Internet of Things to coordinate medical devices) have furnished options for physicians and community health specialists to clearer interpreting personal and public physical variations to detect conditions and provide healthcare, also to improve designing cure and preventive steps. For instance, mobile phones and short messaging service (SMS) can trail health pursuing habits and quantify national and private centres' usage throughout an Ebola epidemic [Feng, Grépin and Chunara 2018]. Individuals, particularly youngsters, devote a substantial time using or engaging with various sorts of channels such as the internet as their main reference for medical information [Wartella, Rideout, Montague, Beaudoin-Ryan and Lauricella 2016]. The internet has broadened community health studies ahead of the classical domain [Shin and ShabanNejad 2017]. Mapping internet-based health indexes augment former monitoring tools retrieving data from health repositories and systems. Breakthroughs in social media technologies and smart web-based gadgets and software serve scholars and community health experts in tracking habits, surveying health conditions, spotting epidemics, and community health education and liaison.

\section{Promising Progress in Health AI}

Some studies have emphasized on activities whereby AI can efficiently prove its ability correlated to a human caregiver. Routinely, these activities have distinctly specified inputs and a dual output, which is feasibly verified. For example, in sorting unusual dermatological injuries, the input is an electronic image and the output is a plain dual categorization: malignant or benign. Within these circumstances, investigators merely had to show that AI held outstanding specificity and sensitivity than dermatology specialists when sorting formerly hidden images of biopsy-verified injuries [Esteva et al. 2017]. 
Moreover, computers miss humankind traits like affection and empathy, and hence patients should understand that the physicians themselves are running visits. Moreover, patients are not supposed to believe in AI instantly; a technology wrapped in doubt [Oppenheim 2016]. Thus, AI usually manages crucial activities, yet quite confined in their nature to pass on the main duty of caring to a patient by a human healthcare provider. In this regard, an in progress clinical trial utilizes AI to compute priority areas for head and neck radiation therapy rather promptly and precisely than a human. A therapeutic radiologist is remaining eventually committed to providing the treatment although AI holds a profound back-end function in safeguarding the patient from unsafe radiation [Chu et al. 2016].

However, an only AI application can stand for a huge community and hence it fits optimally scenarios wherever work force capacity is a deficient asset. For example, in some TB-endemic regions, there is a deficiency in radiologists at distant facilities [Hoog et al. 2011]. Utilizing AI, radiographs uploaded from these facilities could be translated through one focal application; a late research reveals that AI properly diagnoses pulmonary TB with a $100 \%$ specificity and $95 \%$ sensitivity [Lakhani and Sundaram 2017]. Moreover, below-equipped jobs whereby patients are encountering disappointing periods of waiting are likewise appealing to AI triaging application [Burgess 2017].

\subsection{The latest Applications of AI in Health}

Beyond just showing superior adequacy, novel systems joining the health arena should attain proper legal permits, merge in on-line procedures, and motivate patients and healthcare practitioners to contribute to this novel approach. These obstacles have prompted a list of rising courses in AI studies and implementation. Though the following illustrated tracks look encouraging, the producing companies should prove the safety and efficacy regarding their techniques by peer-reviewed research.

A principal application of $\mathrm{AI}$ in medicine is to gather, warehouse, settle, and track data. Google- the search titan- has started its AI research branch so-called "DeepMind Health" to mine medical records' data and render reliable and agile medical services. Together with Moorfields Eye Hospital NHS Foundation Trust, they began a collaborative project to enhance optic therapy in 2016 [DeepMind 2016]. Moorfields distributed one million anonymous optic scans and their associated data about the optic status and condition management to DeepMind for examining whereby the technology can serve in investigating these scans.

IBM Watson started its unique application- "Watson for Oncology"- to equip oncologists with evidence-based treatment alternatives. The application holds an exceptional capacity for examining the setting and significance of structured and unstructured data within records and medical notes to decide on a treatment plan. Next, through merging traits of the patient's portfolio beside medical experience and research, the application recognizes likely treatment strategies for a patient [Zauderer et al. 2014]. IBM started a second application named "Medical Sieve" for producing the upcoming "cognitive health assistant" with rational and logical capacities plus a 
medical knowledge spectrum. Medical Sieve can support cardiologists and radiologists in making clinical decisions. The cognitive assistant can examine radiological images to find and identify issues reliably and quickly [Syeda-Mahmood et al. 2016]. Towards the future, radiologists might simply have to see the most complex problems wherever human guidance is inevitable. Furthermore, IBM Watson Health and Microsoft's InnerEye, a diagnosing computer-assisted analyst for medical images, that can immediately recognize illnesses with the eyes and show diseased spots including cancers [Shrivastav 2019].

Zorgprisma Publiek- a Dutch company- investigates insurance companies and hospitals' digital bills by adopting IBM Watson in the cloud to mine the data. They could determine whether a physician, clinic, or hospital produces errors frequently in managing a particular case to assist them in recovering and bypassing patients' additional admission [Mesko 2017].

"Deep Genomics" can recognize patterns in sets of big data concerning clinical records and hereditary information, scanning for deviations and correlations to disease. They are running upon a novel production of computational technologies, which could inform health professionals anything appears inside a cell if the genetic material is modified by native or therapeutic hereditary mutation [Mesko 2017].

Concerning pharmaceuticals advancement, clinical trials need occasionally longer periods and fetch billions of dollars. Racing this up and executing it with costeffectiveness could produce an immense impact approaching present's medicine including how innovations strike daily healthcare. "Atomwise" employs supercomputers to figure treatments out of a molecular structures' database. The company started a virtual exploration for trustworthy, current medications to be redesigned to manage the Ebola virus in 2016. They discovered two medications via the AI technology prediction that could efficiently diminish Ebola pathogenicity. This study, that could have demanded months or years, was accomplished within one day [Atomwise 2015].

Further applications include da Vinci, an AI-derived robot that enables surgeons to operate in narrow spots since a hand could not deal effectively. It minimizes man flaw rates', offers consistency and accuracy dealing with the highly fine operations and hence satisfactorily lowers operational ramifications' rates; Sense.ly, a virtual nurse assistant, operates with ML algorithms where patients data are introduced to assist in monitoring appointments, filling the gaps between physician encounters, managing patients conditions' and suggesting therapies; Babylon Health that enables detecting, investigating and diagnosing viruses; and Careskore, an AI-assisted health calculator, that tracks vitals for a patient anywhere and anticipates the need for hospital admission [Shrivastav 2019].

\section{Moral aspects of Deploying AI in the Medical Practice}


Irrespective the distributed consumption of intelligent technologies in medicine, there are still constraints to implementation. Adopting technology-particularly for diagnostics in the medical context- raises worries regarding expandability, interoperability and consolidating data, privacy, confidentiality, and morals of accumulated electronic data. For instance, incorporating AI techniques in social media analytics disclosed many moral constraints, which may ultimately sabotage personal liberty and privacy and induce stigma [21]. Moreover, patient intricacy is growing with the falling mean lifespan in the US [22]. Boomers are getting old (20\% of the above 65 years of age citizens by 2029) and comorbidities impact $60 \%$ of them and are coupled with doubled physician-patient interactions [23]. Behaviours and culture settings contribute critically to manage those patients and ought to be fundamental elements in technology-based approaches. Additionally, general constraints are state legislation and guidelines on community healthcare data and genetic research, caregiver's beliefs, vigilance and training, information technology adoption, and monetary matters [24].

Nowadays, an AI software may detect dermatological tumour rather precisely than an expert dermatology specialist [Esteva et al. 2017]. Moreover, the software is able to carry that out competently and swiftly, requiring an educated data collection instead of years of personnel demanding and costly clinical training. Although it could seem that it is merely a short period prior clinicians are brought outdated for this sort of techniques, a sharper glance at the function this systems might act in delivering health services is justified to realize their existing qualities, drawbacks, and moral dilemmas. AI, involving natural language processing, machine learning and robotics domains, might be used in nearly all healthcare areas', and the prospective impact on clinical education, biomedical studies and health services' delivery deems countless [Ramesh, Kambhampati, Monson and Drew 2004]. Via AI's dynamic potential to adapt and explore huge medical data collections, it might take a part in detection, clinical decision-making and precision medicine [Amato et al. 2013, Bennett and Hauser 2013, Dilsizian and Siegel 2014]. For instance, AI-driven detection algorithms used in mammograms are providing recommendations to radiology practitioners, facilitating breast cancer diagnosis [Shiraishi, Li, Appelbaum and Doi 2011]. Furthermore, innovative simulated man avatars can interact in significant discussions that possess dimensions in detecting and managing mental illness [Luxton 2014a]. AI programs as well unfold in the clinicians' domain by physical task support applications, robotic prostheses and portable operators helping in telehealth delivery [Riek 2017].

However, this dynamic innovation fosters a new band of moral constraints that should be recognized and addressed because AI techniques retain considerable potential to jeopardize patient choice, confidentiality and security. Notwithstanding, Present-day AI systems' strategy and regulating ethics are hanging back to the advances it has rendered in the medical arena. Although several initiatives to interact in these moral discussions have arisen, the healthcare society stays unaware of the moral difficulties that emerging AI applications may bring [Luxton 2014b, 2016, Peek, Combi, Marin and Bellazzi 2015]. Correspondingly, an expected and valuable debate that could substantially gain from clinicians' perspective, for they soon would probably be engaging with AI in their regular routine. Some of the highly urgent worries voiced in this matter entail dealing with the further threat to patient 
anonymity and secrecy, exploring the lines across the clinician's and computers' duties in managing patients, and adapting the training of next practitioners to dynamically respond to the inevitable transformations in the healthcare field. Furthermore, negotiations on these issues would enhance caregivers and patients' awareness about the part AI could do in medicine, serving stakeholders to formulate a pragmatic impression about AI capabilities. Subsequently, predicting the likely moral shortcomings, finding feasible fixes, and providing strategy guidelines could contribute to professionals accepting AI approaches into their routine, also the patients who get the service. 


\subsection{AI and Terminating Human Touch}

Beside improvements would additionally appear legitimate problems and moral concerns. For instances, the one to blame if an AI system gives wrong predictions or conclusions and the one to consolidate security points and the marketplace response to the rise of AI while it causes some jobs to go incompetent. Driverless vehicles brought the universal dispute respecting the algorithms' conclusions under complicated circumstances. In medicine, this grows a much consequential moral trial. There are now further unsettled inquiries and probably with global public debates, this would explicate since $\mathrm{AI}$ is growing into a reality. AI further holds severe barriers in medicine. Prediction and forecasting are inferred depending on prior patterns encountered in machine learning, however, algorithms could break in unusual situations of medication resistance or adverse reactions wherever there is no preceding model to formulate on. Therefore, AI might not substitute implied knowledge, which could not be systematized efficiently. Despite the progress in data analysis, it ought to strengthen the health practitioners' abilities and is not intended to substitute the established rapport between patients and health professionals. So as to maintain the human touch in health for expanding the management of the appropriate conditions by the most individualized remedies, the following arrangements could be valuable:

- Founding mandated and relevant moral measures in applying AI for the entire medical practice. A related model is the German state who formulated the world's primary moral guidance for producing independent vehicles. The principles insist that human security should ever be preferred above defending assets or animals and evermore enabling the human driver to replace the application's conclusions though the guideline acknowledges that not all moral judgments could be patterned [Maiberg, Rogers and Lunau 2017].

- Training healthcare providers on the fundamental knowledge regarding how AI operates in the health environment to realize how before-mentioned solutions can support their daily work. Whereas AI is not expected to displace medical practitioners, those who work with AI may substitute those who do not.

- Progressively improving AI by assessing each developing level explicitly by autonomous bioethical investigation societies and organizations prior to moving to the subsequent one, to provide an opportunity for charting the probable impediments and to establish fail-safe systems to counter an $\mathrm{AI}$ apocalypse [Shermer 2017]. 
- Obligating the AI technologies producing companies to provide peerreviewed research and transparent dialogue approaching the broad society around the implied gains and hazards from adopting $\mathrm{AI}$ in healthcare.

- Raising patients' awareness of AI to find out its advantages. For instance, using CogniToys that support young kids' cognitive improvement through AI.

- Taking all the required measures by decision-makers at medical foundations, to gauge the impact and the progress of such systems. It is likewise crucial to driving companies to advance affordable AI solutions for making facts out of science fiction promises and adapting $\mathrm{AI}$ as the stethoscope of the future. Respectively, the FDA (Food and Drug Administration) has authorized some AI technologies for clinical purposes [Arterys 2017].

Consequently, it is crucial to weigh AI approaches' gains versus threats. Efficiently incorporating AI techniques in the medicinal practice may enhance the productivity of delivering health services and managing patient standards. Conversely, it is imperative to reduce moral pitfalls of adopting AI that might involve risks to anonymity and secrecy, informed consent, and patient free will, and count for aligning AI with healthcare system. Stakeholders should be invited to adaptively introduce AI applications, probably as a supplement element and not a substitute for a caregiver. Regardless of drawbacks, early AI methods and technologies yet can afford thorough personal medical data and forecast community health hazards, and their medicinal or population health application is presumed to spike dramatically in the horizon

\section{Results}

Assumptions, patterns and approaches from AI are transforming the health scene in community and clinical perspectives and have previously exhibited encouraging outcomes in various applications in medicine. AI shall have far-reaching implications that will transform the healthcare practice, changing caregivers' regular routines and the patient experience. The application of AI in medicine could even reach surprising fields like artistic work, including brand-new perplexities arising from the emergence of thinking automata in previously human hunts. Although AI in healthcare promises exceptional advantages to patients, it correspondingly implies jeopardies to data security, patient safety, and health equity. Consequently, there is a considerable effort to set the conventional moral basis for practicing AI technology productively and securely in medicine examining patient autonomy and privacy, medical education and, etc. 


\section{Conclusions}

Throughout developmental transformation via digital health, conventional healthcare is shifting into equitable cooperation among patients and health providers. Furthermore, from several disruptive technologies, AI has a prominent perspective to encourage this shift by investigating the immense quantities of data recorded by medical organizations and patients in each instant. Through removing the monotonous elements of a caregivers' work, it could drive to paying extra valuable time for patients and enhancing the human touch. However, AI can just accomplish its purpose if it endures an established, secure and effective support in managing patients and reforming health. For allowing AI grow reliably, information technology should be improved and made interoperable and the quality and scope of clinical data must be thoroughly improved as well. The healthcare institutions should also hold strong strategies to afford backup services if technology systems collapse or are breached.

\section{References}

[Amato, López, Peña-Méndez, Vaňhara, Hampl and Havel 2013] Amato, F., López, A., PeñaMéndez, E. M., Vaňhara, P., Hampl, A., Havel, J.: 'Artificial neural networks in medical diagnosis'; Journal of Applied Biomedicine, Vol. 11, No. 2 (2013), pp. 47-58. https://doi.org/10.2478/v10136-012-0031-x

[Arterys 2017] Arterys: 'Arterys Receives FDA Clearance For The First Zero-Footprint Medical Imaging Analytics Cloud Software With Deep Learning For Cardiac MRI'; (2017). Retrieved 6 June 2019, from https://www.prnewswire.com/news-releases/arterys-receives-fdaclearance-for-the-first-zero-footprint-medical-imaging-analytics-cloud-software-with-deeplearning-for-cardiac-mri-300387880.html

[Atomwise 2015] Atomwise: 'Atomwise finds first evidence towards new Ebola treatments'; (2015). Retrieved from https://www.atomwise.com/2015/03/24/atomwise-finds-first-evidencetowards-new-ebola-treatments/

[Bennett and Hauser 2013] Bennett, C. C., Hauser, K.: 'Artificial intelligence framework for simulating clinical decision-making: a Markov decision process approach'; Artificial Intelligence in Medicine, Vol. 57, No. 1 (2013), pp. 9-19.

https://doi.org/10.1016/j.artmed.2012.12.003

[Burgess 2017] Burgess, M.: 'The NHS is trialling an AI chatbot to answer your medical questions'; Wired UK (2017, January 5). Retrieved from https://www.wired.co.uk/article/babylon-nhs-chatbot-app

[Chu, De Fauw, Tomasev, Paredes, Hughes, Ledsam, et al. 2016] Chu, C., De Fauw, J., Tomasev, N., Paredes, B. R., Hughes, C., Ledsam, J., et al.: 'Applying machine learning to automated segmentation of head and neck tumour volumes and organs at risk on radiotherapy planning CT and MRI scans'; F1000Research, Vol. 5 (2016), p. 2104. https://doi.org/10.12688/f1000research.9525.1

[DeepMind 2016] DeepMind: 'Announcing DeepMind Health research partnership with Moorfields Eye Hospital'; (2016). Retrieved 5 June 2019, from 
https://deepmind.com/blog/announcing-deepmind-health-research-partnership-moorfields-eyehospital/

[Dilsizian and Siegel 2014] Dilsizian, S. E., Siegel, E. L.: 'Artificial intelligence in medicine and cardiac imaging: harnessing big data and advanced computing to provide personalized medical diagnosis and treatment'; Current Cardiology Reports, Vol. 16, No. 1 (2014), p. 441. https://doi.org/10.1007/s11886-013-0441-8

[Elenko, Underwood and Zohar 2015] Elenko, E., Underwood, L., Zohar, D.: 'Defining digital medicine'; Nature Biotechnology, Vol. 33 (2015), pp. 456-461. https://doi.org/10.1038/nbt.3222

[Esteva, Kuprel, Novoa, Ko, Swetter, Blau and Thrun 2017] Esteva, A., Kuprel, B., Novoa, R. A., Ko, J., Swetter, S. M., Blau, H. M., Thrun, S.: 'Dermatologist-level classification of skin cancer with deep neural networks'; Nature, Vol. 542, No. 7639 (2017), pp. 115-118. https://doi.org/10.1038/nature21056

[Feng, Grépin and Chunara 2018] Feng, S., Grépin, K. A., Chunara, R.: 'Tracking health seeking behavior during an Ebola outbreak via mobile phones and SMS'; Npj Digital Medicine, Vol. 1, No. 1 (2018), p. 51. https://doi.org/10.1038/s41746-018-0055-Z

[Ginsburg and McCarthy 2001] Ginsburg, G. S., McCarthy, J. J.: 'Personalized medicine: revolutionizing drug discovery and patient care'; Trends in Biotechnology, Vol. 19, No. 12 (2001), pp. 491-496.

[Hoog, Meme, van Deutekom, Mithika, Olunga, Onyino and Borgdorff 2011] Hoog, A. H. V., Meme, H. K., van Deutekom, H., Mithika, A. M., Olunga, C., Onyino, F., Borgdorff, M. W.: 'High sensitivity of chest radiograph reading by clinical officers in a tuberculosis prevalence survey'; The International Journal of Tuberculosis and Lung Disease: The Official Journal of the International Union Against Tuberculosis and Lung Disease, Vol. 15, No. 10 (2011), pp. 1308-1314. https://doi.org/10.5588/ijtld.11.0004

[Lakhani and Sundaram 2017] Lakhani, P., Sundaram, B.: 'Deep Learning at Chest Radiography: Automated Classification of Pulmonary Tuberculosis by Using Convolutional Neural Networks'; Radiology, Vol. 284, No. 2 (2017), pp. 574-582. https://doi.org/10.1148/radiol.2017162326

[Luxton 2014a] Luxton, D. D.: 'Artificial intelligence in psychological practice: Current and future applications and implications'; Professional Psychology: Research and Practice, Vol. 45, No. 5 (2014a), pp. 332-339. https://doi.org/10.1037/a0034559

[Luxton 2014b] Luxton, D. D.: 'Recommendations for the ethical use and design of artificial intelligent care providers'; Artificial Intelligence in Medicine, Vol. 62, No. 1 (2014b), pp. 110. https://doi.org/10.1016/j.artmed.2014.06.004

[Luxton 2016] Artificial intelligence in behavioral and mental health care: San Diego, CA, US: Elsevier Academic Press (2016).

[Maiberg, Rogers and Lunau 2017] Maiberg, E., Rogers, K., Lunau, K.: 'Germany Has Created the World's First Ethical Guidelines for Driverless Cars'; (2017). Retrieved from https://www.vice.com/en_us/article/599wnz/germany-has-created-the-worlds-first-ethicalguidelines-for-driverless-cars

[Mesko 2017] Mesko, B.: 'The role of artificial intelligence in precision medicine'; Expert Review of Precision Medicine and Drug Development, Vol. 2, No. 5 (2017), pp. 239-241. https://doi.org/10.1080/23808993.2017.1380516 
[Meskó, Drobni, Bényei, Gergely and Győrffy 2017] Meskó, B., Drobni, Z., Bényei, É., Gergely, B., Győrffy, Z.: 'Digital health is a cultural transformation of traditional healthcare'; MHealth, Vol. 3 (2017). https://doi.org/10.21037/mhealth.2017.08.07

[Oppenheim 2016] Oppenheim, M.: 'Stephen Hawking has a terrifying warning about Artificial Intelligence and the future of humanity'; (2016, October 20). Retrieved 13 June 2019, from http://www.independent.co.uk/news/people/stephen-hawking-artificial-intelligence-diasterhuman-history-leverhulme-centre-cambridge-a7371106.html

[Peek, Combi, Marin and Bellazzi 2015] Peek, N., Combi, C., Marin, R., Bellazzi, R.: 'Thirty years of artificial intelligence in medicine (AIME) conferences: A review of research themes'; Artificial Intelligence in Medicine, Vol. 65, No. 1 (2015), pp. 61-73. https://doi.org/10.1016/j.artmed.2015.07.003

[Ramesh, Kambhampati, Monson and Drew 2004] Ramesh, A. N., Kambhampati, C., Monson, J. R. T., Drew, P. J.: 'Artificial intelligence in medicine.'; Annals of the Royal College of Surgeons of England, Vol. 86, No. 5 (2004), pp. 334-338. https://doi.org/10.1308/147870804290

[Riek 2017] Riek, L. D.: 'Healthcare Robotics'; Commun. ACM, Vol. 60, No. 11 (2017), pp. 68-78. https://doi.org/10.1145/3127874

[Shaban-Nejad, Lavigne, Okhmatovskaia and Buckeridge 2017] Shaban-Nejad, A., Lavigne, M., Okhmatovskaia, A., Buckeridge, D. L.: 'PopHR: a knowledge-based platform to support integration, analysis, and visualization of population health data'; Annals of the New York Academy of Sciences, Vol. 1387, No. 1 (2017), pp. 44-53. https://doi.org/10.1111/nyas.13271

[Shermer 2017] Shermer, M.: 'Artificial Intelligence Is Not a Threat--Yet'; (2017). https://doi.org/10.1038/scientificamerican0317-77

[Shin, Mahajan, Akbilgic and Shaban-Nejad 2018] Shin, E. K., Mahajan, R., Akbilgic, O., Shaban-Nejad, A.: 'Sociomarkers and biomarkers: predictive modeling in identifying pediatric asthma patients at risk of hospital revisits'; Npj Digital Medicine, Vol. 1, No. 1 (2018), p. 50. https://doi.org/10.1038/s41746-018-0056-y

[Shin and Shaban-Nejad 2017] Shin, E. K., Shaban-Nejad, A.: 'Public Health Intelligence and the Internet: Current State of the Art'; In A. Shaban-Nejad, J. S. Brownstein \& D. L. Buckeridge (Eds.), Public Health Intelligence and the Internet. Cham: Springer International Publishing (2017), pp. 1-17. https://doi.org/10.1007/978-3-319-68604-2_1

[Shiraishi, Li, Appelbaum and Doi 2011] Shiraishi, J., Li, Q., Appelbaum, D., Doi, K.: 'Computer-aided diagnosis and artificial intelligence in clinical imaging'; Seminars in Nuclear Medicine, Vol. 41, No. 6 (2011), pp. 449-462. https://doi.org/10.1053/j.semnuclmed.2011.06.004

[Shrivastav 2019] Shrivastav, A. A.: 'Top 10 AI apps and solutions that are changing the healthcare industry'; (2019, January 5). Retrieved from https://bigdata-madesimple.com/top10-ai-apps-and-solutions-that-are-changing-the-healthcare-industry/

[Syeda-Mahmood, Walach, Beymer, Gilboa-Solomon, Moradi, Kisilev, et al. 2016] SyedaMahmood, T., Walach, E., Beymer, D., Gilboa-Solomon, F., Moradi, M., Kisilev, P., et al.: 'Medical sieve: a cognitive assistant for radiologists and cardiologists'; In Medical Imaging 2016: Computer-Aided Diagnosis (Vol. 9785). International Society for Optics and Photonics (2016), p. 97850A. https://doi.org/10.1117/12.2217382

[Wartella, Rideout, Montague, Beaudoin-Ryan and Lauricella 2016] Wartella, E., Rideout, V., Montague, H., Beaudoin-Ryan, L., Lauricella, A.: 'Teens, Health and Technology: A National 
ICEAT 2019

Survey'; Media and Communication, Vol. 4, No. 3 (2016), pp. 13-23. https://doi.org/10.17645/mac.v4i3.515

[Zauderer, Gucalp, Epstein, Seidman, Caroline, Granovsky, et al. 2014] Zauderer, M. G., Gucalp, A., Epstein, A. S., Seidman, A. D., Caroline, A., Granovsky, S., et al.: 'Piloting IBM Watson Oncology within Memorial Sloan Kettering's regional network.'; Journal of Clinical Oncology, Vol. 32, No. 15_suppl (2014), pp. e17653-e17653. https://doi.org/10.1200/jco.2014.32.15_suppl.e17653 